\title{
Original
}

\section{Sealing ability of mineral trioxide aggregate (MTA) combined with distilled water, chlorhexidine, and doxycycline}

\author{
Roberta A. A. Arruda1), Rodrigo S. Cunha'), Kenner B. Miguita1), \\ Cláudia F. M. Silveira1), Alexandre S. De Martin1), Sérgio L. Pinheiro3), \\ Daniel G. P. Rocha ${ }^{1,3)}$ and Carlos E. S. Bueno ${ }^{1,3)}$ \\ 1)Department of Endodontics, São Leopoldo Mandic School of Dentistry and Dental Research Center, \\ Campinas, SP, Brazil \\ 2)Department of Restorative Dentistry, University of Manitoba, Winnipeg, MB, Canada \\ ${ }^{3)}$ Department of Endodontics, Pontifical Catholic University of Campinas, Campinas, SP, Brazil
}

(Received 28 February and accepted 10 July 2012)

\begin{abstract}
The aim of this study was to evaluate the sealing ability of mineral trioxide aggregate (MTA Bio) combined with different mixing agents (distilled water, chlorhexidine, doxycycline), used as an apical root-end filling material. Forty-two extracted human teeth were divided into three groups $(n=12)$; six teeth were used as controls. Root-ends were resected at 90 degrees, $3 \mathrm{~mm}$ from the apex. Root-end cavities were prepared using ultrasonic tips and filled with MTA Bio plus distilled water, $2 \%$ chlorhexidine solution, or $10 \%$ doxycycline solution. Apical sealing was assessed by microleakage of $50 \%$ silver nitrate solution. Roots were longitudinally sectioned in a buccolingual plane and analyzed using an operating microscope (20 $\times$ magnification). Depth of dye leakage into the dentinal walls was measured in millimeters. Results were analyzed using ANOVA and Tukey's test $(P=$ 0.05). MTA Bio plus distilled water showed significantly higher mean leakage results $(1.06 \mathrm{~mm})$ when compared with MTA Bio plus doxycycline $(0.61 \mathrm{~mm})$, and higher, although not significant, results when compared with MTA Bio plus chlorhexidine (0.79 $\mathrm{mm})$. In conclusion, replacing distilled water with
\end{abstract}

Correspondence to Dr. Roberta Aranha de Araujo Arruda, Rua Barão de Atibaia, 789, CEP 13023-011, Campinas, SP, Brazil Tel: $+55-19-3252-5355$

Fax: +55-19-3251-5656

E-mail: roberta.aranha@terra.com.br two biologically active mixing agents (doxycycline and chlorhexidine) did not alter the sealing properties of MTA Bio. The antimicrobial properties of these combinations should be further investigated. (J Oral Sci 54, 233-239, 2012)

Keywords: apical microleakage; mineral trioxide aggregate; silver nitrate; root-end filling material.

\section{Introduction}

Different materials are used for root-end filling, including gutta-percha, amalgam, zinc oxide-eugenol (ZOE) cement, CAVIT, composite resin, glass ionomer cement, reinforced ZOE cement (Super EBA) and mineral trioxide aggregate (MTA). Of these, MTA has been used to seal communications between the root canal system and the external tooth surface (1). MTA was first recommended as a root-end filling material when developed, but it has been used for pulp capping procedures, pulpotomy, apexogenesis, apical barrier formation in teeth with open apexes, repair of root perforations, and as a root canal filling material (2).

MTA promotes hard tissue deposition and presents excellent biocompatibility. Many clinicians consider it as the gold-standard material for use in apical surgery (3). Previous studies comparing MTA with other rootend filling materials such as amalgam, Super EBA, and Intermediate Restorative Material (IRM) have reported 
significantly less dye and bacterial leakage and better adaptation associated with MTA $(4,5)$. Koçak et al. (6) established MTA as the preferred root-end filling material for use with different techniques, based on its increased sealing ability. In addition, Rosales-Leal et al. (7) obtained a hermetic seal with Clearfil and MTA due to the excellent marginal fit and recommended these materials for clinical use, taking into consideration their sealing capacity.

The antimicrobial properties of MTA have also been extensively evaluated, with conflicting results $(5,8)$. Several investigations reported that MTA has limited antimicrobial effect against some microorganisms $(5,9)$. One of these studies demonstrated that MTA had an antibacterial effect on some facultative bacteria, but no effect on any of the strict anaerobes (4). Another antimicrobial study assessed the antimicrobial effect of MTA, Portland cement, Sealapex, ZOE against a mixture of Staphylococcus aureus, Enterococcus faecalis, Pseudomonas aeruginosa, Bacillus subtilis, and Candida albicans. Sealapex and ZOE did not show microbial leakage over the experimental period, whereas MTA and Portland cement showed leakage between 15 and 45 days (5). In an agar diffusion test under anaerobic conditions, inhibition zones were observed in white- and gray-colored MTA cements over Escherichia coli mutants and triple mutants (8). Al-Hezaimi et al. (10) showed that both white- and gray-colored MTA cements at different concentrations were able to inhibit the growth of $C$. albicans and $E$. faecalis.

A Brazilian company (Angelus Indústria de Produtos Odontológicos Ltd, Londrina, PR, Brazil) recently developed white- and gray-colored MTA cements (WhiteMTA-Angelus and Gray-MTA-Angelus) and also a new formulation, MTA Bio, for different clinical applications in dental practice. According to the manufacturer, MTA Bio is fully synthesized under highly controlled laboratory conditions to ensure that the final product is free of undesirable contaminants, such as arsenic and lead. Due to its high purity and low solubility, MTA Bio is claimed to have low toxic effects on pulp cells, allowing faster and more effective tissue healing after direct pulp capping or pulpotomy (11). De-Deus et al. (12) demonstrated that White-MTA-Angelus, MTA Bio and Portland cement presented a similar ability to seal furcal perforations in extracted human molars. Moreover, Lessa et al. (11) observed low cytotoxic effects of White-MTA and MTA Bio on odontoblast-like cell cultures (MDPC-23).

Endodontic filling materials can be applied with mixing agents to improve their antimicrobial activity. Chlorhexidine was introduced as an irrigant in the early 1960s, and since then has been considered an effective antimicrobial agent against Enterococcus faecalis in vitro (13). Holt et al. (14) assessed the antimicrobial effect of chlorhexidine against E. faecalis and the compressive strength of a combination of MTA and chlorhexidine $2 \%$ in comparison with MTA mixed with sterile water, and found better results with the former combination. Similarly, doxycycline, a hydroxy derivative of tetracycline, is the most potent anticollagenase antibiotic among the tetracyclines commercially available (15). The antimicrobial properties of doxycycline were better than those of calcium hydroxide when used as an intracanal medication as it was capable of eliminating the bacterial biofilm inside human tooth canals (16).

In spite of the promising evidence available and the substantivity (i.e., long-term residual antimicrobial activity) shared by both chlorhexidine and doxycycline (17), no study so far has been designed to determine whether a combination of these two substances with MTA would enhance the antimicrobial properties of this filling material.

Therefore, the objective of the present study was to evaluate, ex vivo, the root-end sealing ability of MTA Bio (Angelus, Londrina, PR, Brazil) combined with either distilled water, $2 \%$ chlorhexidine solution, or $10 \%$ doxycycline solution, employed as root-end filling materials, using a dye penetration test with silver nitrate solution.

\section{Materials and Methods}

\section{Sample selection}

The present study protocol was reviewed and approved by the Research Ethics Committee of São Leopoldo Mandic School of Dentistry and Dental Research Center, Campinas, Brazil (protocol no. 2009/016).

Forty-two freshly extracted single-rooted human maxillary central incisors with fully formed apices were selected. Following previously established protocols $(5,18)$, preoperative mesiodistal and buccolingual radiographs of each root were taken to verify that only one root canal was present and that there was no internal or external resorption or calcification. To avoid dehydration, teeth were stored in $0.9 \%$ sodium chloride solution (19).

All specimens were examined under an operating microscope (DF Vasconcellos, São Paulo, SP, Brazil) at $20 \times$ magnification to exclude teeth with cracks or fractured apices. Selected teeth were sectioned at the cementoenamel junction using a low-speed diamond saw (KG Sorensen Ltd., Cotia, SP, Brazil) under continuous water spray to create a standardized root length of about $16 \mathrm{~mm}$. 


\section{Root canal treatment}

Working length was established by placing a $21-\mathrm{mm}$ \#10 Kerr file (Dentsply Maillefer, Ballaigues, Switzerland) in the canal until its tip became visible at the apical foramen, and subtracting $1 \mathrm{~mm}$. Canals were shaped using the crown-down technique with ProTaper rotary files (Dentsply Maillefer). The following technical sequence was used during coronal preparation: \#10, \#15, S1, Sx, working length, \#15, S1, and S2. F1, F2, F3, and F4 were used as master apical files (MAF) to facilitate the delivery of endodontic irrigants.

During instrumentation, root canals were irrigated with $5 \mathrm{~mL}$ of freshly prepared $5.25 \%$ sodium hypochlorite $(\mathrm{NaOCl})$ solution and rinsed with $3 \mathrm{~mL}$ of $17 \%$ ethylenediaminetetraacetic acid (EDTA) for $5 \mathrm{~min}$ to remove the smear layer. After cleaning and shaping, each canal was dried with sterile paper points (Dentsply Maillefer) and obturated using Tagger's hybrid technique with guttapercha and Grossman root canal sealer. Each root-filled tooth was restored with a provisional dressing (Coltosol, Colténe, Altstatten, Switzerland). The teeth were stored in $100 \%$ humidity for $48 \mathrm{~h}$ to prevent fracture during the cutting process (20).

\section{Root-end preparation}

An apical resection was made 90 degrees to the longitudinal axis of each tooth, $3 \mathrm{~mm}$ away from the root apex, using a water-cooled, high-speed diamond bur (KG Sorensen Ltd.). Root-end cavities of $3 \mathrm{~mm}$ were prepared using an ultrasonic ENAC ST37-90 tip (Osada, Tokyo, Japan).

\section{Root-end filling}

Following apical preparation, teeth were randomly divided into three groups ( $n=12$ each) according to the root-end filling material: Group 1, MTA Bio $0.12 \mathrm{~g}$ (Angelus Soluções Odontológicas, Londrina, PR, Brazil) combined with $90 \mu \mathrm{L}$ of distilled water; Group 2, MTA Bio $0.12 \mathrm{~g}$ combined with $90 \mu \mathrm{L}$ of $2 \%$ chlorhexidine solution; and Group 3, MTA Bio $0.12 \mathrm{~g}$ combined with $90 \mu \mathrm{L}$ of $10 \%$ doxycycline solution. Six teeth were used as controls, as follows: positive controls $(n=3)$, teeth not receiving any root-end filling material; and negative controls $(n=3)$, teeth receiving MTA Bio $0.06 \mathrm{~g}$ combined with $45 \mu \mathrm{L}$ of distilled water.

MTA Bio was prepared using $0.12 \mathrm{~g}$ of MTA Bio powder and $90 \mu \mathrm{L}$ of the mixing agents employed in each group, in order to guarantee adequate consistency of the final product. The powder:liquid ratio recommended by the manufacturer (Angelus) for MTA Bio is 3:1. However, based on a pilot study, the ratio was modified $(0.12 \mathrm{~g}$ of powder to $90 \mu \mathrm{L}$ of liquid) in order to ensure a consistent mixture throughout the study.

Filling materials were applied using a MAP (MicroApical Placement) System (Roydent Dental Products, Johnson City, TN, USA), and a retro filling endodontic plugger \# 1 (Hu-Friedy, Chicago, IL, USA) was used to condense MTA Bio in root-end cavities.

Two layers of nail polish, each one with a different color, were applied to the whole surface of each root, except for the apex. Teeth in the negative control group $(n=3)$ had the whole surfaces protected by nail polish (including the apex).

\section{Apical microleakage}

Teeth were immersed in 50\% silver nitrate solution and kept in the dark, at room temperature, for $2 \mathrm{~h}$. Subsequently, they were rinsed under running distilled water for $1 \mathrm{~min}$ to remove silver ions adhering to the surface, immersed in a photo-developing solution (Dektol, Kodak, Brazil), and exposed to light for $12 \mathrm{~h}$. Teeth were washed once again in distilled water, and roots were longitudinally sectioned in a buccolingual plane.

Longitudinal grooves were cut on the buccal and lingual root surfaces using a \#3071 diamond bur. Grooves were engraved as deeply as possible (close to the root canal walls) using a double-face diamond disk. A cement spatula $\# 24 \mathrm{~F}$ was placed in the groove, and the root was longitudinally sectioned in two halves using a slow-speed diamond saw. The half presenting the best conditions for visual analysis was removed from the cavity and subsequently used for analysis. All samples were randomly numbered and photographed using a Sony PC 120 camera coupled to an operating microscope at $20 \times$ magnification. Images were analyzed using the Image Tool software version 3.0.

One single examiner, blinded to group allocation, assessed all dye penetration tests on Image Tool 3.0 (single blind testing). The depth of silver nitrate penetration was recorded with the aid of a millimeter ruler photographed together with the specimens. In order to allow the examiner to accurately assess leakage depth, the root-end filling material was removed from the canals (21). A straight line was marked from the most apical point of the penetrated area to the deepest point at the opposing end of the infiltrate; leakage depth was recorded in millimeters.

\section{Statistical analysis}

Results were statistically analyzed using one-way analysis of variance (ANOVA) to assess the presence of significant differences across the groups in terms of 
Table 1 One-way ANOVA results for the mean leakage depths observed in different groups

\begin{tabular}{lcccc}
\hline Groups & Mean & Standard deviation & 95\% confidence interval & Tukey's test* \\
\hline Group 1 (distilled water) & 1.0600 & 0.4510 & $1.3465-0.7735$ & $\mathrm{~A}$ \\
Group 2 (chlorhexidine) & 0.7908 & 0.2990 & $0.9808-0.6009$ & $\mathrm{~A}, \mathrm{~B}$ \\
Group 3 (doxycycline) & 0.6050 & 0.3640 & $0.8363-0.3737$ & $\mathrm{~B}$ \\
\hline
\end{tabular}

*Different letters indicate significant differences according to the Tukey's test $(P=0.05)$.

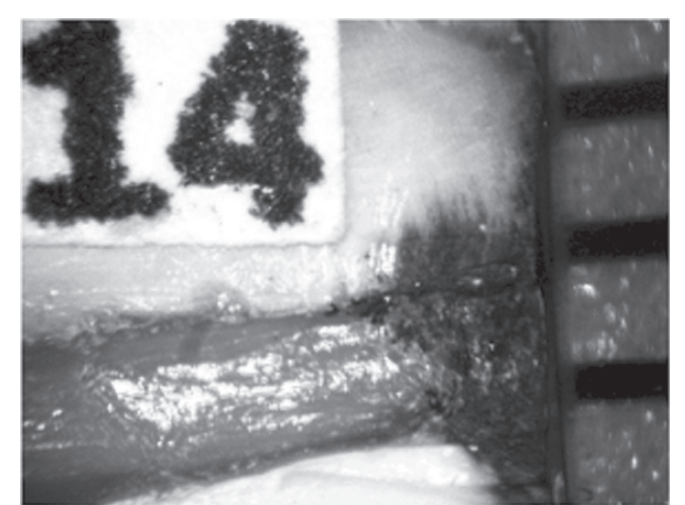

Fig. 1 Specimen from Group 1, treated with MTA Bio combined with distilled water (20× magnification).

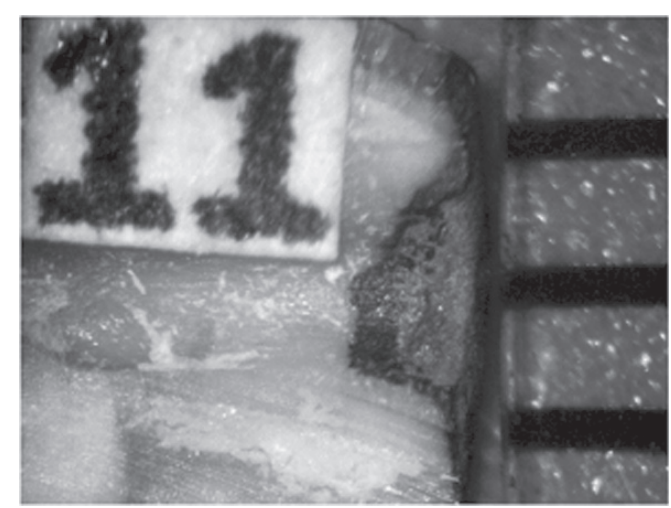

Fig. 2 Specimen from Group 2, treated with MTA Bio combined with $2 \%$ chlorhexidine solution (20× magnification).

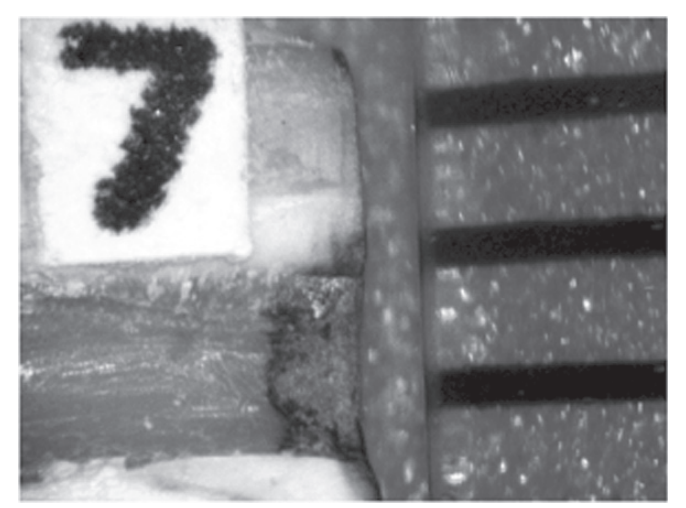

Fig. 3 Specimen from Group 3, treated with MTA Bio combined with $10 \%$ doxycycline solution (20× magnification). dye penetration depth. Tukey's test was used to compare the means obtained in specific pairs of groups (pairwise comparison). Significance was set at $P=0.05$.

\section{Results}

Table 1 shows mean and standard deviation microleakage results obtained in each group. Mean leakage was higher in Group 1 (MTA Bio + distilled water $=1.06 \mathrm{~mm}$ ) when compared with Groups 2 (MTA Bio + chlorhexidine $=0.79 \mathrm{~mm}$ ) and $3($ MTA Bio + doxycycline $=0.61 \mathrm{~mm})$. Figures 1, 2, and 3 show examples of leakage results observed in the different study groups.

ANOVA revealed statistically significant differences between the groups. The Tukey's test, used for pairwise comparison, showed significant differences $(P=0.05)$ between Groups 1 and 3 only (Table 1). Negative controls did not present any leakage, whereas positive controls showed full dye penetration.

\section{Discussion}

The present study compared the sealing ability of MTA Bio combined with different mixing agents, namely distilled water, 2\% chlorhexidine solution, and $10 \%$ doxycycline solution. Root-end filling materials must provide adequate marginal seal; this justifies the present investigation. Both MTA Bio and MTA are biocompatible materials (11). However, MTA contains arsenic and lead, whereas MTA Bio is produced under strict quality control procedures and does not include these substances (manufacturer information) (22). Previous in vitro studies comparing the sealing ability of different root-end filling materials have shown superior results for MTA when compared with others, e.g., amalgam, IRM, Super EBA, and Vitremer (19,21).

Several methodologies have been proposed to assess apical microleakage associated with different root-end filling materials. Among such methodologies, dye penetration has long been used in view of its simplicity and because no additional chemical reactions are required to identify leakage $(18,20)$. Several dyes have been used to determine apical leakage, e.g., methylene blue, eosin, crystal violet, Indian ink, rhodamine $\mathrm{B}$, and silver nitrate (18,21). Methylene blue was applied as a dyeing agent 
for the assessment of sealing ability. However, when in contact with alkaline substances, such as MTA, this dye induces discoloration, affecting leakage depth measurement (23). This limitation has caused methylene blue to be gradually replaced with other dyes.

In our study, silver nitrate was used as the dyeing agent, according to the methodology proposed by Xavier et al. (19) and Costa et al. (18). This dye presents improved definition and allows a more accurate assessment of leakage when compared with other substances; moreover, its use is compatible with optical microscopy (24). However, dye microleakage studies also have some limitations; the leakage can only be measured in one plane. As a result, it is impossible to accurately assess the total amount of leakage $(20,25)$.

One of the main factors influencing the results of microleakage studies is vapor lock (air bubbles) in the root canal (26), which prevents dye penetration. Bubbles of air trapped in voids along the root canal can be removed either by centrifugation or a vacuum machine (25). Roda and Gutmann (27) did not find significant differences in dye penetration after using different leakage evaluation tests, with or without reduced air pressure methods. Based on these findings, we decided not to use a vacuum machine in our study.

The selection of materials for use in association with MTA Bio was based on the antimicrobial action and substantivity of chlorhexidine and doxycycline. Both were used in commercially available liquid formulations, so as to standardize their use and the concentrations necessary to guarantee effective antimicrobial action.

Chlorhexidine is a disinfecting agent that is effective against a wide range of microorganisms, including Enterococcus faecalis (14). MTA can be mixed with chlorhexidine rather than with water when used as a retrofilling material, provided its sealing ability and biocompatibility are confirmed (28). Stowe et al. (29) have demonstrated that MTA shows improved antimicrobial properties when mixed with $0.12 \%$ chlorhexidine instead of water.

Doxycycline, a member of the tetracycline antibiotic group, is one of the components of MTAD (it is responsible for the antibacterial action of this irrigant) (17). For this reason, we decided to combine doxycycline with MTA Bio to analyze the sealing ability of this newly formed material. No previous reports of this combination were found in the literature.

The three material combinations assessed in the present study allowed the dyeing solution to penetrate into apical structures, which is in accordance with previous findings $(1,19)$. No statistically significant differences were observed between distilled water and chlorhexidine. This finding is compatible with the results reported by Shahi et al. (28), who did not observe differences in the sealing ability of gray and white MTA mixed with water or $0.12 \%$ chlorhexidine. They suggested that mixing MTA with chlorhexidine does not affect the sealing ability of the material. In the present study, in turn, statistically significant differences were found between MTA Bio plus doxycycline vs. MTA Bio plus distilled water. This difference in sealing ability can probably be explained by the substance used in this combination, that is, doxycycline. Furthermore, the apparently shorter setting time in Group 3 and the more friable aspect of this newly formed compound when compared with Group 1 could also account for the differences.

Similar findings were reported by Stowe et al. (29), who replaced sterile water with $0.12 \%$ chlorhexidine gluconate as the mixing agent and found that MTA plus chlorhexidine seemed to set more rapidly than MTA plus water and it took on a more crumbly texture at placement. Holt et al. (14) also reported that the combination of MTA plus chlorhexidine was extremely brittle and showed very low compressive strengths when compared with sterile water. Therefore, the combined use of MTA and chlorhexidine would probably not be indicated for clinical situations in which the material is subject to mastication forces.

Because the newly formed compounds in the present study were used for root-end filling, their low resistance to fracture did not contraindicate their use. Previous studies designed to compare these newly formed compounds have assessed only their antimicrobial activity $(14,29)$. To the best of the authors' knowledge, no study has assessed apical microleakage associated with use of MTA plus doxycycline and chlorhexidine.

Further studies should be carried out to assess not only sealing ability but also several other properties of the new compounds (e.g., physical properties, biocompatibility, dimensional stability, antimicrobial property, cytokine production, cytotoxicity, genotoxicity). Moreover, these materials need to be tested under controlled conditions before they can be recommended for clinical application.

Our results and the methodology herein employed allow us to conclude that the replacement of distilled water with two biologically active agents, doxycycline and chlorhexidine, did not affect the sealing ability of MTA Bio. Further research is warranted to assess the antimicrobial properties of these combinations.

\section{References}

1. Aqrabawi J (2000) Sealing ability of amalgam, 
super EBA cement, and MTA when used as retrograde filling materials. Br Dent J 188, 266-268.

2. Parirokh M, Torabinejad M (2010) Mineral trioxide aggregate: a comprehensive literature review - Part I: chemical, physical and antibacterial properties. J Endod 36, 16-27.

3. Pistorius A, Willershausen B, Briseño Marroquin B (2003) Effect of apical root-end filling materials on gingival fibroblasts. Int Endod J 36, 610-615.

4. Torabinejad M, Hong CU, Pitt Ford TR, Kettering JD (1995) Antibacterial effects of some root end filling materials. J Endod 21, 403-406.

5. EstrelaC,Estrada-BernabéPF, deAlmeida-Decurcio D, Almeida-Silva J, Rodrigues-Araújo-Estrela C, Poli-Figueiredo JA (2011) Microbial leakage of MTA, Portland cement, Sealapex and zinc oxideeugenol as root-end filling materials. Med Oral Patol Oral Cir Bucal 16, e418-424.

6. Koçak MM, Koçak S, Aktuna S, Görücü J, Yaman SD (2011) Sealing ability of retrofilling materials following various root-end cavity preparation techniques. Lasers Med Sci 26, 427-431.

7. Rosales-Leal JI, Olmedo-Gaya V, VallecilloCapilla M, Luna-del-Castillo JD (2011) Influence of cavity preparation technique (rotary vs. ultrasonic) on microleakage and marginal fit of six end-root filling materials. Med Oral Patol Oral Cir Bucal 16, e185-189.

8. Ribeiro CS, Scelza MF, Hirata Júnior R, Buarque de Oliveira LM (2010) The antimicrobial activity of gray-colored mineral trioxide aggregate (GMTA) and white-colored MTA (WMTA) under aerobic and anaerobic conditions. Oral Surg Oral Med Oral Pathol Oral Radiol Endod 109, e109-112.

9. Yasuda Y, Kamaguchi A, Saito T (2008) In vitro evaluation of the antimicrobial activity of a new resin-based endodontic sealer against endodontic pathogens. J Oral Sci 50, 309-313.

10. Al-Hezaimi K, Al-Shalan TA, Naghshbandi J, Simon JH, Rotstein I (2009) MTA preparations from different origins may vary in their antimicrobial activity. Oral Surg Oral Med Oral Pathol Oral Radiol Endod 107, e85-88.

11. Lessa FCR, Aranha AMF, Hebling J, Souza Costa CA (2010) Cytotoxic effects of White-MTA and MTA-Bio cements on odontoblast-like cells (MDPC-23). Braz Dent J 21, 24-31.

12. De-Deus G, Reis C, Brandão C, Fidel S, Fidel RA (2007) The ability of Portland cement, MTA, and MTA Bio to prevent through-and-through fluid movement in repaired furcal perforations. J Endod
33, 1374-1377.

13. Siqueira JF Jr, Rôças IN, Santos SR, Lima KC, Magalhães FA, de Uzeda M (2002) Efficacy of instrumentation techniques and irrigation regimens in reducing the bacterial population within root canals. J Endod 28, 181-184.

14. Holt DM, Watts JD, Beeson TJ, Kirkpatrick TC, Rutledge RE (2007) The anti-microbial effect against Enterococcus faecalis and the compressive strength of two types of mineral trioxide aggregate mixed with sterile water or $2 \%$ chlorhexidine liquid. J Endod 33, 844-847.

15. Davis JL, Jeansonne BG, Davenport WD, Gardiner D (2003) The effect of irrigation with doxycycline or citric acid on leakage and osseous wound healing. J Endod 29, 31-35.

16. Saber Sel-D, El-Hady SA (2012) Development of an intracanal mature Enterococcus faecalis biofilm and its susceptibility to some antimicrobial intracanal medications; an in vitro study. Eur J Dent 6, 43-50.

17. Tong Z, Zhou L, Kuang R, Lv H, Qu T, Ni L (2012) In vitro evaluation of MTAD and nisin in combination against common pathogens associated with root canal infection. J Endod 38, 490-494.

18. Costa AT, Post LK, Xavier CB, Weber JBB, Gerhardt-Oliveira M (2008) Marginal adaptation and microleakage of five root-end filling materials: an in vitro study. Minerva Stomatol 57, 295-300.

19. Xavier CB, Weismann R, de Oliveira MG, Demarco FF, Pozza DH (2005) Root-end filling materials: apical microleakage and marginal adaptation. J Endod 31, 539-542.

20. Shahi S, Yavari HR, Rahimi S, Eskandarinezhad M, Shakouei S, Unchi M (2011) Comparison of the sealing ability of mineral trioxide aggregate and Portland cement used as root-end filling materials. J Oral Sci 53, 517-522.

21. Torabinejad M, Higa RK, McKendry DJ, Pitt Ford TR (1994) Dye leakage of four root end filling materials: effects of blood contamination. J Endod 20, 159-163.

22. Vivan RR, Zapata RO, Zeferino MA, Bramante CM, Bernardineli N, Garcia RB, Hungaro Duarte MA, Tanomaru Filho M, Gomes de Moraes I (2010) Evaluation of the physical and chemical properties of two commercial and three experimental root-end filling materials. Oral Surg Oral Med Oral Pathol Oral Radiol Endod 110, 250-256.

23. Tanomaru Filho M, Figueiredo FA, Tanomaru JM (2005) Effect of different dye solutions on the 
evaluation of the sealing ability of mineral trioxide aggregate. Braz Oral Res 19, 119-122.

24. Holt GM, Dumsha TC (2000) Leakage of amalgam, composite, and Super-EBA, compared with a new retrofill material: bone cement. J Endod 26, 29-31.

25. Tamse A, Katz A, Kablan F (1998) Comparison of apical leakage shown by four different dyes with two evaluating methods. Int Endod J 31, 333-337.

26. Wu MK, De Gee AJ, Wesselink PR (1994) Fluid transport and dye penetration along root canal filling. Int Endod J 27, 233-238.

27. Roda RS, Gutmann JL (1995) Reliability of reduced air pressure methods used to assess the apical seal. Int Endod J 28, 154-162.

28. Shahi S, Rahimi S, Yavari HR, Shakouie S, Nezafati S, Abdolrahimi M (2007) Sealing ability of white and gray mineral trioxide aggregate mixed with distilled water and $0.12 \%$ chlorhexidine gluconate when used as root-end filling materials. J Endod 33, 1429-1432.

29. Stowe TJ, Sedgley CM, Stowe B, Fenno JC (2004) The effects of chlorhexidine gluconate $(0.12 \%)$ on the antimicrobial properties of tooth-colored ProRoot mineral trioxide aggregate. J Endod 30, 429-431. 